Delft University of Technology

\title{
Learning geothermal energy basics with the serious game hotpipe
}

Mac An Bhaird, Liam; Al Owayyed, Mohammed; van Driel, Ronald; Jiang, Huinan; Johannessen, Runar A.; Salamon, Nestor Z.; Balint, J. Timothy; Bidarra, Rafael

DOI

10.1007/978-3-030-34350-7_30

Publication date

2019

Document Version

Final published version

Published in

Games and Learning Alliance

\section{Citation (APA)}

Mac An Bhaird, L., Al Owayyed, M., van Driel, R., Jiang, H., Johannessen, R. A., Salamon, N. Z., Balint, J. T., \& Bidarra, R. (2019). Learning geothermal energy basics with the serious game hotpipe. In A. Liapis, G. N. Yannakakis, M. Gentile, \& M. Ninaus (Eds.), Games and Learning Alliance: 8th International Conference, GALA 2019, Proceedings (Vol. 11899, pp. 312-321). (Lecture Notes in Computer Science (including subseries Lecture Notes in Artificial Intelligence and Lecture Notes in Bioinformatics); Vol. 11899 ). Springer Science+Business Media. https://doi.org/10.1007/978-3-030-34350-7_30

Important note

To cite this publication, please use the final published version (if applicable).

Please check the document version above. 
Green Open Access added to TU Delft Institutional Repository 'You share, we take care!' - Taverne project

\section{https://www.openaccess.nl/en/you-share-we-take-care}

Otherwise as indicated in the copyright section: the publisher is the copyright holder of this work and the author uses the Dutch legislation to make this work public. 


\title{
Learning Geothermal Energy Basics with the Serious Game HotPipe
}

\author{
Liam Mac an Bhaird, Mohammed Al Owayyed, Ronald van Driel,
} Huinan Jiang, Runar A. Johannessen, Nestor Z. Salamon, J. Timothy Balint, and Rafael Bidarra ${ }^{(凶)}$

Faculty of Electrical Engineering, Mathematics and Computer Science, Delft University of Technology, Delft, The Netherlands

R.Bidarra@tudelft.nl

\begin{abstract}
Burning fossil fuels is a big part of our heat production. Since this process is both non-renewable and polluting, finding other options is important. A clean and underutilized alternative is geothermal energy. However, it is often not considered due to sheer ignorance or misconceptions. HotPipe is a serious game designed to alleviate these problems, particularly among youth populations. Players control a drill to create geothermal wells solving a variety of puzzles, which introduce relevant cases for geothermal heating and show what geothermal wells are made of. The game focuses primarily on conveying the concepts of water circulation, relation between temperature and depth, and rock type proprieties. From our game evaluation, players revealed a solid improvement on their geothermal energy knowledge.
\end{abstract}

Keywords: Renewable energy $\cdot$ Geothermal heating $\cdot$ Doublet $\cdot$ Drilling $\cdot$ Puzzle $\cdot$ Serious games $\cdot$ Sandstone

\section{Introduction}

According to figures published by Energie Beheer Nederland [9], heat consumption accounts for nearly $40 \%$ of overall primary energy consumption in the Netherlands. This consumption is mainly produced by burning fossil fuels, particularly natural gas. Conventionally, conversion to renewable energy would reduce the established adverse effects of burning fossil fuels [15]. Geothermal energy is regarded as a suitable alternative to fossil fuels [19] as it is eco-friendly, economical in the long term, and efficient. Geothermal systems take advantage of the earth's heat by extracting it from the subsurface. Heat extraction occurs by using pipes to transfer fluids (e.g., water) from subsurface geothermal reservoirs to the surface and vice-versa. Common geothermal reservoirs are aquifers, porous ground layers that contain hot fluids. In order to achieve the transfer, a cycle with two wells is needed: one to obtain hot water and the other to inject the water after the heat has been extracted [13]. Such production wells are assumed to last 30 years and require approximately 100 years to regenerate [1].

(C) Springer Nature Switzerland AG 2019

A. Liapis et al. (Eds.): GALA 2019, LNCS 11899, pp. 312-321, 2019.

https://doi.org/10.1007/978-3-030-34350-7_30 
We introduce HotPipe, a serious game that aims at conveying the basic concepts of geothermal heating systems, thus countering ignorance and misconceptions in this domain. Its target audience is the youth, particularly senior high school and undergraduate students. HotPipe is a 2D puzzle game in which the player implements a geothermal system by drilling through a tile-based maze representing the Earth's subsurface, to connect pipes to the underground water reservoirs. A screenshot of one of the early game levels is shown in Fig. 1. Specifically, the game aims at conveying the knowledge of:

- water circulation mechanisms of a geothermal well;

- positive correlation between temperature and depth in the subsurface;

- some rock type properties influence drilling speed; and

- sandstone is the preferable rock type for a water reservoir.

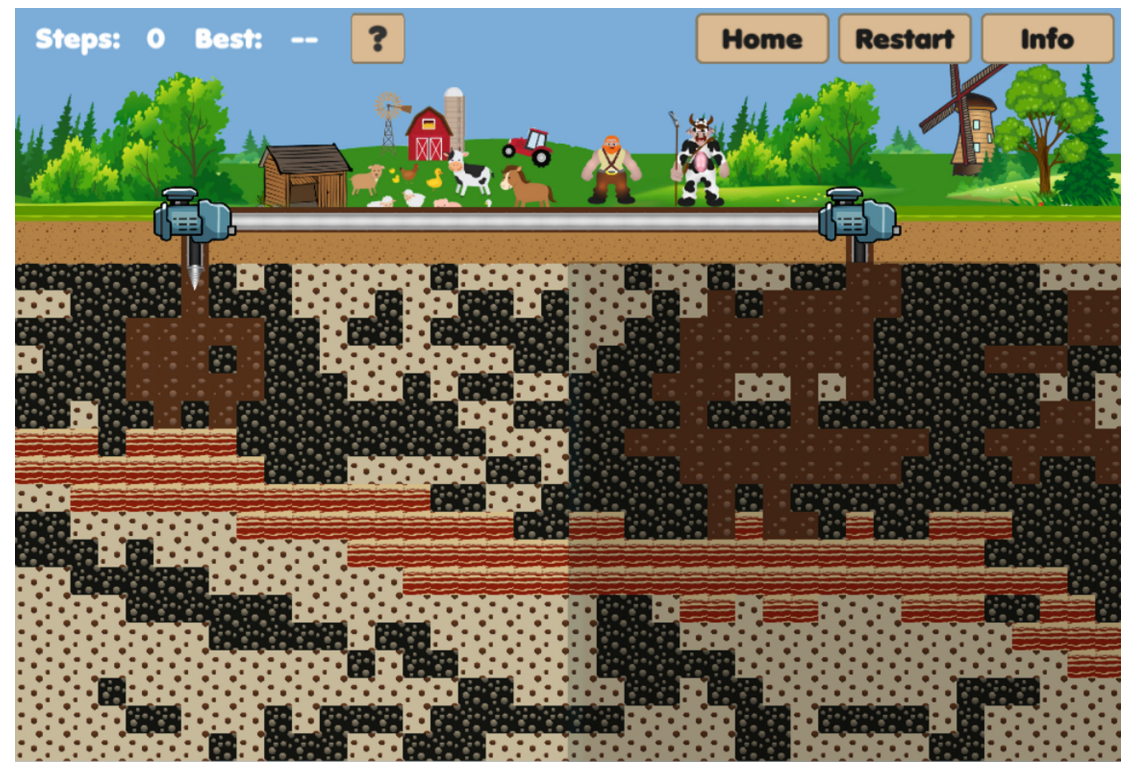

Fig. 1. HotPipe: screen capture of an early game level

\section{Related Work}

So far, there have been a few proposals to apply gamification techniques to teach geothermal systems to children. Environment for Kids: Geothermal Energy [17] is a post-lesson quiz aiming to measure children's understanding of geothermal energy and the knowledge they gained from lessons. The Groundwater Term 
Game [8] takes this concept one step further by providing a more interactive method for answering questions.

To the best of our knowledge, HotPipe is the first serious game about geothermal energy. However, there have been a few other serious games with some remote similarities. Brasil et al. designed a serious 3D game to train operators in oil drilling fields [6]. The game allows operators to exercise their knowledge and practice handling troublesome situations. MAEGUS is a serious game developed to advocate for sustainable energy [16]. Similar to the oil-drilling game, MAEGUS provides players with real-life situations related to a city's energy consumption plans. A player, being a city planner, must adhere to the plans by providing renewable energy to the city (such as geothermal and wind energy).

Several commercially available games have integrated geothermal energy into their gameplay. An example is Turmoil [4], in which the player takes the role of an oil exploration company to gain profit and develop a town. The game simplifies drilling concepts while focusing more on the financial aspects of running an oil extraction company. As for its drilling-puzzle mechanics, Hotpipe can be compared to Motherload [5]. The main concept behind Motherload is to drill through rock layers and collect the scattered rare minerals by controlling a robot-like machine. However, Motherload focuses on exploration and resource management rather than on puzzle solving as in HotPipe. Pipe-based games tend to have the same idea of linking point A to point B (e.g., Line Puzzle [3], Flow Free [2] and Water Pipes [14]). However, these games depend on connecting various pipes on a $2 \mathrm{D}$ surface rather than combining them with drilling in different layers.

\section{Game Design}

In this section we focus on how HotPipe is designed to engage the players, as well as on how its game mechanics guides them towards the essential concepts, including the rock types and the water flow.

\subsection{Story Setting}

In HotPipe you are a friendly dwarf named Flint who is out to solve other NPCs' (non-playable characters') heating problems using geothermal systems. Each level is split into two puzzles representing the two core elements of a geothermal system: an injection well and a production well, which we refer to as a level pair. A water flow animation is played when both wells are complete and the NPC is able to generate a sufficient amount of heat. The setting is designed this way to not only engage with the mechanics of the game but also have an emotional connection to the characters. This gives the player a purpose to complete each level and the reward of seeing more water flow animations. 


\subsection{Game Mechanics}

HotPipe is a tile-based puzzle game. Most people have played puzzle games, so the game is intuitive and does not distract from the educational goals. The player's goal is to create a geothermal well by drilling into the sandstone. The game provides a map with locations for a drilling site just as it would in real life. Next to this, there is also an info page which helps link the game elements to real world applications in the context of geothermal systems. Game elements on the info page contain items such as characters, rock types, geothermal instruments, as well as a tab dedicated to the general workings of a geothermal system. Additionally, the game allows the player to complete level pairs as many times as possible so as to reinforce the educational aspect. The player is further exposed to the general workings of a geothermal system through the level pair complete (water flow) animation.

Controls. The controls in HotPipe are straightforward and intuitive. The arrow controls move the drill in the direction of the key press, and an undo key allows for safe exploration. There is, however, one 'less intuitive' movement, which is key to making HotPipe a unique puzzle experience: the default move (for shale and sandstone) is 2 tiles. This is something that the player must grow familiar with in the early levels. This is also the game mechanics that puts the player in awkward situations. Wonky controls are not only healthy for puzzle games (such as Portal [21] and Snakebird [18]) but, in this case, also represent that in the real world the driller does not have full control over their drill on certain soils.

Rock Tiles. HotPipe features five rock types: shale, granite, limestone, rock salt and sandstone. The behaviour of the different rock types is explained in Table 1. Each level was designed using tiles of these 5 rock types. Aside from these, there are also pipe tiles and a drill head tile. Drilling can be blocked by pipe tiles, granite tiles and limestone tiles.

Progression. Progression in HotPipe is the result of successive puzzle completion. New levels are unlocked progressively as puzzles are completed. Bonus levels are optional, and have increased difficulty, represented with a trickster NPC so the player knows to expect more of a challenge.

Rewards. In-game rewards are based on the amount of steps it takes for the player to complete the level. When players complete a puzzle, they earn either 1, 2 or 3 Golden Dabbing Dwarfs (GDDs), depending on how close to optimal their solution is. This is an addition which represents a cost of inefficiency in the geothermal world. The player receives a reward for completing all non-bonus levels with an end-of-game sequence. Nonetheless, challenging puzzles are very rewarding in themselves to complete. Our careful level design makes the challenge interesting and fair so as to maximize this reward. 
Table 1. Rock type tiles and their behavior in HotPipe.

\begin{tabular}{|l|l|l|}
\hline Rock tile & Rock type & Behavior in the game \\
\hline Sandstone & $\begin{array}{l}\text { Winning condition, players succeed when they drill to the } \\
\text { andstone tiles (in some levels, multiple layers are used, where } \\
\text { digging deeper encounters a higher quality sandstone layer). }\end{array}$ \\
\hline Rock salt & $\begin{array}{l}\text { Very soft rock and is drilled through very quickly in real life. } \\
\text { This is exaggerated in the game and lets the drill move forward } \\
\text { until there is no more rock salt. }\end{array}$ \\
\hline Shale & Medium softness rock. The drill moves two steps through these tiles. \\
\hline
\end{tabular}

\subsection{Visuals}

The art style is purposely simple to put focus on the game mechanics and concepts. The tile set is simplified to a single texture for each rock type. This makes different rock types distinguishable albeit less realistic, as our goal is not to teach the player what the rock types look like, but rather to explain their differences in relation to geothermal drilling.

The most important visual element is the animation showing how water flows in a geothermal system, which plays after the player completes a geothermal well (drilling a level pair). This water flow is a core element to the basic workings of geothermal systems. The steam effect animation clearly illustrates that the water being extracted is hotter than the water being pumped down the well (see Fig. 2). This is repeated for each completed well, in an effort to consistently expose players to this essential concept.

\section{Level Design}

The main reward mechanic of most puzzle games is the actual puzzle. The puzzle must be challenging enough for the player to struggle a little but still end up learning how to complete the puzzle. Unfortunately the amount of challenge that a puzzle provides is very subjective. Therefore, having a target audience makes it easier to design for a specific group in mind. With the target audience of HotPipe, puzzles need to be slightly more challenging than for young children, as this group is assumed to have been exposed to puzzle games before, making the learning process quicker.

The set of tools used for the puzzle design follows the Gamemaker's toolkit principles [10]. In HotPipe, the level design tools align with the following aspects: 


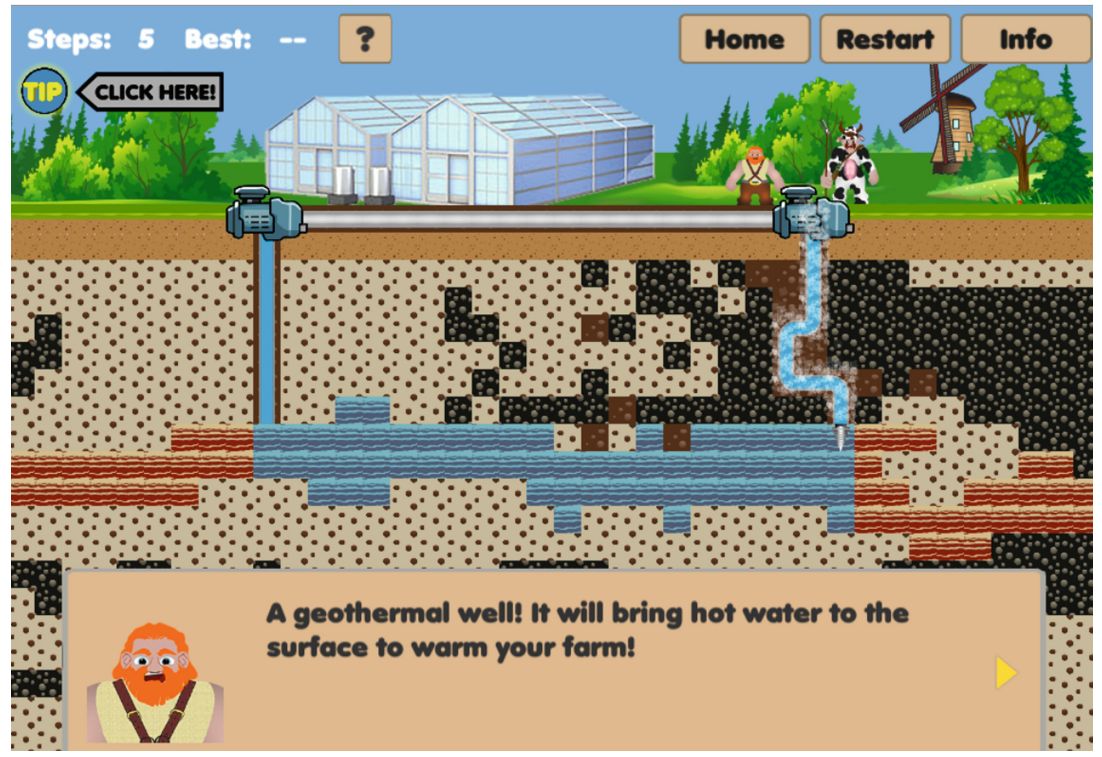

Fig. 2. Water flowing through the system, upon level completion

- The catch: a logical contradiction that the player encounters (e.g., turn right to go left).

- The revelation: an understanding that the player acquires in order to solve the puzzle (e.g., use an impassable rock to limit further drilling).

- The assumption: an assumption can be added to guide a player into the wrong direction, preferably guiding the player into a catch. This assumption must be broken by the player to solve the puzzle (e.g., the player is led to loop around some element).

For the first three levels, the puzzles build upon each other in an introductory fashion, to both retain players and teach them the main workings of the game. Overall, the simplicity of the first levels helps the player focus just on the most relevant game aspects.

Level 1 is an introductory level and is thus kept as simple as possible. Therefore, it only teaches the player the controls and the levels goal.

Level 2 guides the player to the corner of a small corridor. Once the player reaches this corner, they get stuck as there remains no other option but to overshoot the exit, as shown in Fig. 3. Here, they are presented with the catch in that the pipe moves two tiles at a time through this type of rock. This is reinforced if they restart the level and move left. The revelation occurs when they understand that the drill moves two tiles on shale and this is the reason they get trapped leading to the solution shown in Fig. 3.

Level 3 explore the knowledge gained on previous levels. Here the player has already been exposed to the common mechanics of overshooting an exit. Now 

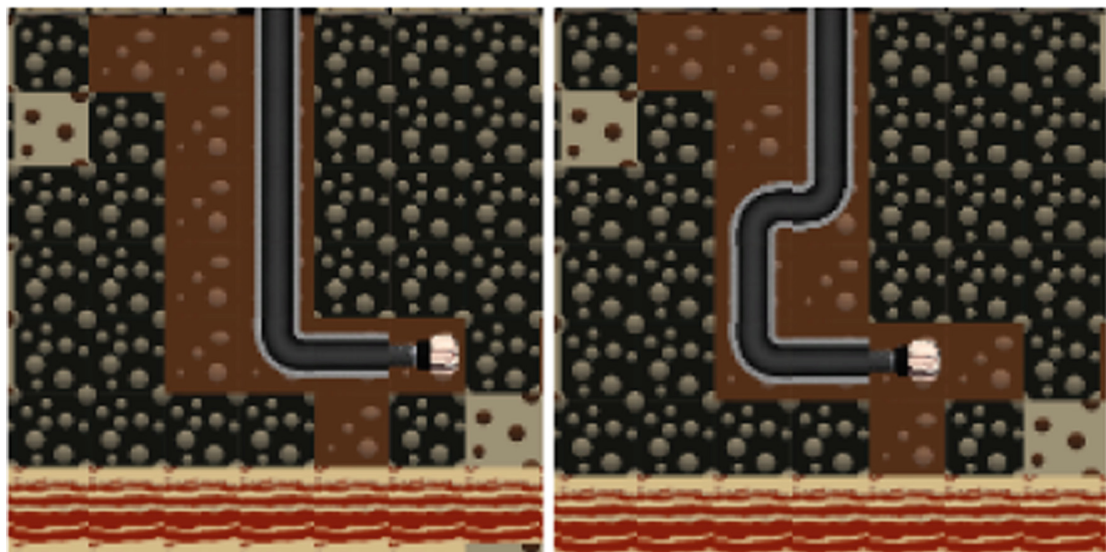

Fig. 3. A player overshooting the exit (left) and the solution learned (right)
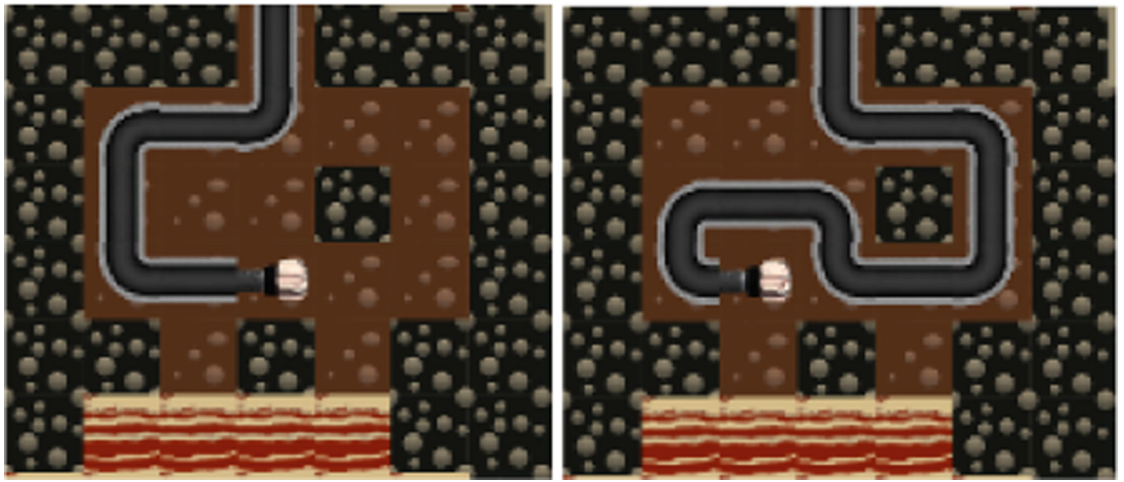

Fig. 4. The player is guided into the revelation that their own pipe can block unwanted drilling progressing.

they only need to be introduced to the not-so-intuitive but common mechanic of using their own pipe to help reach the exit. Again the player is presented with the overshoot problem (Fig. 4). The catch is, this time, they do not have any granite walls that they can use to reach the exit. The player is guided to the solution through: (i) use of minimal possible directions to move at each point, and (ii) the block in the middle of the level, which has no influence on the puzzle's solution other than removing symmetry, but still invites the player to loop around it and further reduces move choice.

After the first three levels, which work as tutorials, the player should be familiar with the controls, the main game mechanics, as well as have experienced two catches. Over the subsequent 17 levels, the player should be able to cope with the increased complexity. 


\section{Implementation}

The game was developed using Unity [20]. Initial level design and testing was done using Puzzlescript [12], an online free-access platform for quickly creating and testing $2 \mathrm{D}$ puzzle games.

The game implementation was kept data-oriented, using the same data structure for loading different maps, level backgrounds and character dialogues. For user interface, we implemented window pop-up, fade in, zoom in and scaling effect to enhance players' game feel. The water flow animation in game was done using sprite sequence animation, which simulates the water going through pipes. We also designed particle effects for breaking stones and water steam.

The biggest challenge was the puzzle design. The skill and experience needed to complete each puzzle should not be perceived as too difficult nor too easy. As such it was important that the difficulty started at an accessible level and increased at a good pace to keep the game challenging yet surmountable.

Some technical limitations arose due to the game being 2D and tile-based, the largest one being the simplifications in relation to reality. The tile-based mechanics dictates player movement in that it allows for simple gameplay mechanics and makes puzzle creation easier. On the other hand, it leads the player to drill in an unrealistic fashion by taking sharp turns and not necessarily going straight for the sandstone. While the game is not intended to be a realistic drilling simulator, we did not want to give players a false impression that drilling operations are a simple endeavor. Thus, the info page explains how wells are created in real life and what it requires of planning and calculation to drill correctly.

\section{Evaluation}

We tested the game with 23 volunteers recruited from TU Delft campus, being 16 volunteers within the audience-targeted range of 15-20 years, and 7 above that range. We designed our evaluation primarily to assess the extent to which the main geothermal concepts and message were captured after playing the game. The participants were asked to play seven levels. On average, they spent two minutes solving introductory levels, and 15 min solving final puzzles.

According to Breuer et al. [7], people tend to learn more if an educational game is engaging. In order to measure the knowledge conveyed by the game, we applied Bloom's taxonomy of educational objectives [11] by defining goals based on the hierarchical levels of the cognitive domain. From the aforementioned domain, the learning goals of HotPipe cover the first three levels: knowledge (i.e. remembering learned information), comprehension (i.e. understanding the meaning), and the ability to use concepts in a new situation, referred to as application. We prepared a brief pre- and post-survey for the participants asking two questions about geothermal systems-related topics (see Fig. 5) to roughly estimate their knowledge before and after playtesting the game. Both surveys had similar questions, but were displayed in randomized order to avoid bias. 


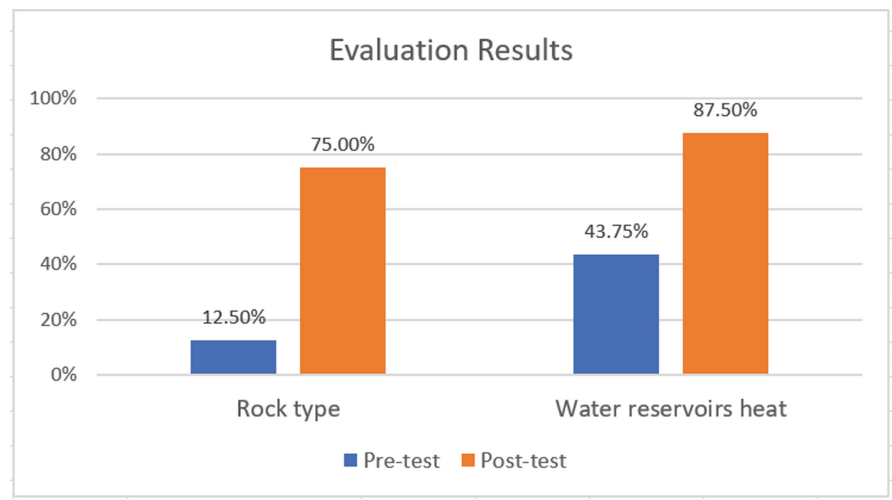

Fig. 5. Percentage of correct answers in pre- and post-surveys.

\subsection{Results}

The 7 adult participants already had reasonable knowledge about geothermal systems, and thus had little to learn from HotPipe. We therefore focused our analysis on the remaining 16 participants on the target group. Their performance on the surveys increased significantly after playing the game (Fig. 5).

\subsection{Discussion}

Overall, all participants liked the game and endorsed the controls; most of them described it as easy and intuitive. Almost all the participants figured out the game mechanics by solving the puzzles in order. They did struggle with the last bonus puzzle, but referred to that level as being challenging rather than frustrating, which is a positive indication. We noticed that participants who answered the "rock type" question incorrectly remembered the textures of the rocks. Therefore, we decided to add more elements to the game that display the rock names when hovering over them with a mouse or when a new rock type is introduced in a new level. These elements do not constrict the flow of the puzzles, yet expose the player to rock names more frequently.

\section{Conclusions}

HotPipe is an intuitive and attractive puzzle game, smoothly integrating education with an enjoyable experience to enhance the appeal of learning. The game focuses on geothermal system fundamentals, including various operations such as drilling and water transfer. As such, HotPipe provides a good introduction to geothermal systems for the target audience. We believe this may be also true for younger groups, due to the atmosphere of friendly competition created.

In the future, we would like to increase the number of levels, as new ideas are still appearing, and some others were put aside for being overly frustrating. 
Nonetheless, several other promising geothermal features would deserve further work, including: a store where players can buy drill heads with different properties, a seismic truck for viewing the underground map and ground stimulation techniques.

HotPipe is free to play and is available at: https://hotpipe.itch.io/hotpipe.

Acknowledgement. The authors thank Richard R. Bakker for his helpful suggestions on many geothermal energy-related concepts presented in the game.

\section{References}

1. Banwell, C.J.: Life expectancy of geothermal fields. Geother. Energy 2(7), 12-13 (1974)

2. Big Duck Games LLC: Flow free. http://tiny.cc/ilzocz

3. Bitmango: Line puzzle. http://tiny.cc/vpzocz

4. Bouman, J., Leppen, E.: Turmoil. http://tiny.cc/vrzocz

5. Boyes, S.: Motherload. http://www.xgenstudios.com/play/motherload

6. Brasil, I.S., et al.: An intelligent and persistent browser-based game for oil drilling operators training. In: 2011 IEEE 1st International Conference on Serious Games and Applications for Health (SeGAH), pp. 1-9. IEEE (2011)

7. Breuer, J., Bente, G.: Why so serious? On the relation of serious games and learning. J. Comput. Game Culture 4, 7-24 (2010)

8. Eirich, M.: The groundwater term game. http://tiny.cc/qxzocz

9. Energie Beheer Nederland B.V. (EBN): Energy in the Netherlands (2016). http:// www.energieinnederland.nl/2016

10. Game Maker's Toolkit: What makes a good puzzle? https://youtu.be/zsjC6fa_YBg

11. Krathwohl, D.R., Anderson, L.W.: A Taxonomy for Learning, Teaching, and Assessing: A Revision of Bloom's Taxonomy of Educational Objectives. Longman (2009)

12. Lavelle, S.: Puzzlescript! https://www.puzzlescript.net/

13. Lund, J., Sanner, B., Rybach, L., Curtis, R., Hellstrom, G.: Geothermal (groundsource) heat pumps-a world overview. Geo-Heat Cent. Q. Bull. 25(3) (2004)

14. Mobiloids: Water pipes. http://tiny.cc/8ozocz

15. Munack, A., Schröder, O., Krahl, J., Bünger, J.: Comparison of relevant exhaust gas emissions from biodiesel and fossil diesel fuel. Agr. Eng. Int.: CIGR J. Sci. Res. Dev. Manuscript EE 01001 III (2001). https://cigrjournal.org/index.php/ Ejounral/article/view/195

16. Nataraja, K., Whittinghill, D.: MAEGUS: a sustainable energy simulation to promote energy literacy. In: E-Learn: World Conference on E-Learning in Corporate, Government, Healthcare, and Higher Education (2013)

17. Nelson, K.: Environment for kids: geothermal energy. http://tiny.cc/q2zocz

18. Noumenon Games: Snakebird (2015). http://tiny.cc/kzzocz

19. Omer, A.M.: Ground-source heat pumps systems and applications. Renew. Sustain. Energ. Rev. 12(2), 344-371 (2008)

20. Unity Technologies: Unity game engine-official site (2019). http://unity.com

21. Valve: Portal. https://store.steampowered.com/app/400/Portal/ 